\title{
Effects of Supplemental Feeding of Probiotics during Lactation on Rumen Microflora of Calves after Weaning
}

\author{
Xueyan Lin, Tian Zhang, Lin Ju, Yue Jiang, Qiuling Hou, Zhiyong Hu, Yun Wang, Zhonghua Wang* \\ College of Animal Science, Shandong Agricultural University, Tai'an, China \\ Email: linxueyan@sdau.edu.cn, *zhwang@sdau.edu.cn
}

How to cite this paper: Lin, X.Y., Zhang, T., Ju, L., Jiang, Y., Hou, Q.L., Hu, Z.Y., Wang, Y. and Wang, Z.H. (2021) Effects of Supplemental Feeding of Probiotics during Lactation on Rumen Microflora of Calves after Weaning. Advances in Bioscience and Biotechnology, 12, 213-228.

https://doi.org/10.4236/abb.2021.127014

Received: June 3, 2021

Accepted: July 19, 2021

Published: July 22, 2021

Copyright $\odot 2021$ by author(s) and Scientific Research Publishing Inc. This work is licensed under the Creative Commons Attribution International License (CC BY 4.0).

http://creativecommons.org/licenses/by/4.0/

(c) (i) Open Access

\begin{abstract}
For the experiment, 8 newborn male Holstein calves were selected that had the same feeding environment, and were of similar ages. They were randomly divided into 2 groups, with 4 in each group. The treatments consisted of feeding active probiotics (Group P) and a normal fed control group (Group C). The growth performance and blood indices were measured; rumen fluid samples were collected after weaning, and 16SrDNA sequencing and LC-MS metabolome detection were performed. Compared with the control group, relative abundances of Deltaproteobacteria, Desulfovibrionales, Bacteroidales BS11_gut_group, Desulfovibrionaceae, Bacteroidales_S24-7_group, Acetobacteraceae, Ruminococcaceae_NK4A214_group, Asaia, [Ruminococcus] gauvreauii_group, Desulfovibrio, Kingella, Selenomonas, Lachnoclostridium in group $\mathrm{P}$ were significantly different $(\mathrm{P}<0.05)$. In group $\mathrm{P}$, the metabolite of 2 -methylbenzoic acid and myo-inositol were significantly increased $(\mathrm{P}<0.05)$. These results showed that compared with normally fed calves, the growth performance and blood indices of probiotic-fed calves were changed, but the differences were not significant. Probiotic-fed calves showed significant differences in rumen fluid and a small number of metabolites, which were mainly involved in the pathway of carbohydrate metabolism. It proves that the supplemental active probiotics had an effect on the rumen microflora.
\end{abstract}

\section{Keywords}

Calves, Probiotics, Rumen Fluid, Serum, Metabolites

\section{Introduction}

The concept of probiotics may have originated from the theory first proposed by the Nobel laureate Russian scientist Metchnikoff. He suspected that the longevi- 
ty of Bulgarian farmers is due to fermented dairy products [1]. The term probiotics was originally coined by Lilly to describe substances that one microbe secretes to promote the growth of another [2]. Parker subsequently proposed that probiotics contribute to intestinal microbial balance [3]. Fuller defines probiotics as living microbial feed additives that beneficially affect host animals by improving microbial balance. He also mentioned that probiotics are biological agents containing living cells or stable local microbial metabolites, which may optimize the colonization and composition of the intestinal flora of animals and humans and may support digestion and host immunity. Probiotics are defined as non-pathogenic microorganisms, which have a positive effect on the health or physiology of the host when ingested. They restore and maintain the balance of ideal microorganisms during stress or disease, and promote the growth of young animals [4]. Probiotics are viable microorganisms, and when given in sufficient numbers as probiotics, can alter the microbiota of the host's digestive tract [5], thereby improving health and production. Probiotics are now widely used as feed additives in livestock and have been defined as non-pathogenic microorganisms. They aim to improve productivity and disease prevention by maintaining a healthy gastrointestinal environment and improving the intestinal tract [6]. Probiotics enhance the rumen microbial ecosystem [7], nutrient digestibility [8], nutrient absorption and feed conversion, thus enabling animals to have better production performance. In newborn calves, the microflora is susceptible to changes in diet and environment. Weaning is one of the challenges of young animals. At this stage, calves experience physiological, nutritional and environmental challenges that can easily lead to microfloral imbalances. Bacterial probiotics antagonize the growth of pathogenic bacteria by producing a variety of inhibitory substances against gram-positive and gram-negative pathogenic bacteria. Potential inhibitors may include organic acids, hydrogen peroxide, and bacteriocins. In addition, many lactic acid bacteria produce antibiotic metabolites (acidophillin, acidolin, lactobacilli, and lactocidin), which inhibit Salmonella, Shigella, Staphylococcus, Proteus, Klebsiella, Pseudomonas, Bacillus, and Vibrio Activity, inhibition of enteropathogenic E. coli. Probiotics exert immunomodulatory effects by stimulating the immune system [9]. Probiotics can improve the production of immunoglobulins [10] and enhance the activity of macrophages and natural killer cells [11]. They also modulate the production of antiinflammatory and proinflammatory cytokines [12]. Probiotics have an antagonistic effect on adhesion sites and pathogenic microorganisms of vegetative growth factors [13], which can reduce the risk of intestinal infections [14]. Probiotics have been found to enhance host immunity by stimulating the production of immunoglobulin, macrophages, natural killer cells and cytokines. However, the exact mechanism by which probiotics exert their beneficial effects has not been fully elucidated.

The digestive enzyme system of young ruminants is not well developed. The protease system is not fully established after birth, pepsin is produced late, and the ability to digest solid materials such as foodstuffs is weak. Bacillus subtilis is nonpathogenic and secretes a variety of antibiotics and enzymes. Studies have 
shown that Bacillus subtilis can produce proteases, cellulase, $\alpha$-amylase, phytase, $\beta$-glucanase, xylanase, pectinase and other enzymes [15]. Bacillus subtilis can also produce vitamin $\mathrm{K}$, pig blood peptides [16], $\beta$-mannanase, cyclic dipeptides [17], and antibacterial peptides [18]. These active substances have significant inhibitory effects on pathogenic bacteria. The Bacillus subtilis spores are ideal for processing into microcapsule packets for introduction into calves, as they are highly stable: they are resistant to oxidation, compression, long term temperatures of $60^{\circ} \mathrm{C}$, acids and alkali. They can remain active in the acidic stomach environment, resisting the attack of saliva and bile, allowing $100 \%$ of live bacteria to reach the large intestine.

Yeast can promote the growth and enzymatic activity of cellulolytic bacteria, improve microbial protein synthesis and fiber digestibility [8]. They have high vitamin and protein content and can be used as both food source and for medicinal proposes. It is also possible to extract cytochrome $\mathrm{C}$, nucleic acids, glutathione, coagulation, coenzyme A and adenosine triphosphate. Beneficial substances such as yeast metabolites and cell walls lead to balanced gastrointestinal microflora [19] and relieve stress [20]. The yeast Saccharomyces cerevisiae can produce many growth factors such as vitamin B, branched chain fatty acids, amino acids and peptides. The addition of Saccharomyces cerevisiae cultures to dairy cow diets is effective in stabilizing the rumen fermentation environment [21].

\section{Materials and Methods}

\subsection{Test Design}

For the experiment, 8 newborn Holstein male calves were selected that had the same feeding environment, and were of similar ages. They were randomly divided into 2 groups, with 4 in each group. In the control group, the calves were fed milk + diet (Group C); the experimental group calves were fed milk + diet + compound probiotics (P group). The probiotics were initiated in the second week, and $10 \mathrm{~g}$ was supplemented at each feeding for one week duration. Probiotics are composed of Bacillus subtilis and Saccharomyces cerevisiae. Bacillus subtilis $(\mathrm{CFU} / \mathrm{g}) \geq 9.0 \times 10^{7}$, Saccharomyces cerevisiae $(\mathrm{CFU} / \mathrm{g}) \geq 1.0 \times 10^{7}$, moisture $(\%) \leq 45$.

\subsection{Calves Feeding Management}

After birth the calves' umbilical cords were soaked in a solution of $7 \%-10 \%$ of iodine, and they were weighed. Colostrum was Pasteurized and provided to calves at $36^{\circ} \mathrm{C}$ to $40^{\circ} \mathrm{C}$. Calves were fed $4 \mathrm{~L}$ of high-quality colostrum in one hour ( $8 \%-10 \%$ of body weight), and $3-4 \mathrm{~L}$ of colostrum in $6-8$ hours to ensure a total intake of 6 - $8 \mathrm{~L}$ colostrum within 12 hours. Normal milk was fed 8 hours after feeding colostrum, and feeding took place twice daily (7:00 and 15:00). Calves unable to drink milk properly were fed using a bottle. On the 4th day of birth, the feeding method was changed to milk bucket feeding. Milk was 
provided throughout the day. The calves were freely supplied with water starting on the third day after birth. Warm water was provided in winter, and water was available 24 hours. Calves were fed daily according to a standard (Table 1). All management and experimental procedures were conducted according to the Laboratory Animals Guideline of welfare and ethics of China.

\subsection{Sample Collection and Processing}

\section{Feed Intake Record}

During the test period, the amount of feed and the amount of feed remaining were accurately recorded each day.

\section{Weight and Body Size Measurement}

Body size measurements were taken by the same person to ensure consistency.

Body height: The vertical distance from the highest point of the withers to the ground, as measured with a measuring stick.

Body length: the distance from the shoulder to the end of the ischial bone, as measured with a tape measure.

Chest circumference: The vertical circumference of the body at the posterior horn of the shoulder blade, as measured with a tape measure.

Cannon circumference: The circumference measured at one third of the forelimb cannon bone, as measured with a tape.

Body weight was measured before and after the test period.

\section{Blood Collecton and Sample Preparation}

Blood was collected from the jugular vein and approximately $20 \mathrm{~mL}$ of blood was collected per calf. The blood collection time was $7 \mathrm{am}$, and blood was taken in the morning from 15 and 60 days old calves. After the blood was collected, it was placed in a coagulation tube and allowed to stand at room temperature for $30 \mathrm{~min}$. When the serum was precipitated, it was centrifuged at $3000 \mathrm{r} / \mathrm{min}$ for $15 \mathrm{~min}$ in a low-speed centrifuge, and the serum was aspirated with a pipette and dispensed into a $1.5 \mathrm{~mL}$ centrifuge tube and stored at $-20^{\circ} \mathrm{C}$.

\section{Collection of Rumen Fluid}

After the tenth week, calves were slaughtered and the rumen fluid was squeezed from the rumen chyme, filtered through four layers of gauze, placed in a $5 \mathrm{~mL}$ cryotube, and stored at $-80^{\circ} \mathrm{C}$ until testing.

\subsection{Sample Analysis}

Analysis of Microbial Diversity in Rumen Fluid Based on 16s Rdna V3 + V4 Region

After extracting the total DNA of the sample, Primers were designed to anneal

Table 1. Amount of milk fed to the calves.

\begin{tabular}{ccccccc}
\hline \multicolumn{8}{c}{ Days } \\
\hline & $1-10$ & $11-20$ & $21-30$ & $31-40$ & $41-50$ & $51-55$ \\
\hline Milk & $4.5 \mathrm{~L}$ & $6.5 \mathrm{~L}$ & $8 \mathrm{~L}$ & $8.5 \mathrm{~L}$ & $4 \mathrm{~L}$ & $3 \mathrm{~L}$ \\
\hline
\end{tabular}


to conserved regions, with sequencing primer homology on their 5' ends. Total DNA was extracted and PCR amplification was carried out. PCR products were purified, quantified and homogenized to form a sequencing library, and the library was sequenced by Illumina HiSeq 2500. The sequences were clustered at a level of similarity of $97 \%$.

\section{Metabolomics Nanlysis of Rumen Fluid Based on Lc-Ms}

Samples were analyzed with the Agilent 1290 UHPLC Ultra High Liquid Meter and AB 5600 Triple TOF Mass Spectrometer. The data were analyzed by statistical methods using orthogonal projections to latent structures-disciplinant analysis (OPLS-DA). Differential metabolites were screened by combining the $\mathrm{P}$ value of the Student's $t$ test with the VIP value of the OPLS-DA model. The criteria for screening were $\mathrm{P}$ value $<0.05$ and VIP $>1.5$.

\section{Data Analysis}

The raw data was statistically processed using EXCEL. The data was analyzed using the One-Way ANOVA model in SAS 8.2 software and compared using Duncan's multiple test. $\mathrm{P}<0.01$ indicates that the difference is highly significant, $0.01<\mathrm{P}<0.05$ indicates that the difference is significant, and $0.05<\mathrm{P}<0.1$ indicates that there is a tendency to differ. The result is expressed in the form of means \pm SEMs.

\section{Result}

\subsection{Growth Performance Result}

Table 2 shows the effects of supplemental probiotics on the growth and feed intake of calves. It can be seen from the table that there was no differences between the two treatment groups before the test $(\mathrm{P}>0.05)$. After supplementing the

Table 2. Effects of rumen fluid supplementation on growth performance of calves.

\begin{tabular}{|c|c|c|c|}
\hline \multicolumn{3}{|c|}{ Group } & \multirow{2}{*}{$\mathrm{p}$} \\
\hline & $\mathrm{C}$ & $\mathrm{P}$ & \\
\hline \multicolumn{4}{|l|}{ Before the treatment } \\
\hline Weight, kg & $40.38 \pm 1.16$ & $38.13 \pm 2.70$ & 0.47 \\
\hline Height, cm & $77.93 \pm 2.23$ & $76.65 \pm 2.31$ & 0.71 \\
\hline Length, cm & $68.00 \pm 1.91$ & $69.23 \pm 1.92$ & 0.67 \\
\hline Chest girth, $\mathrm{cm}$ & $79.00 \pm 0.91$ & $77.50 \pm 1.66$ & 0.46 \\
\hline \multicolumn{4}{|l|}{ After the treatment } \\
\hline Weight, kg & $95.25 \pm 3.33$ & $98.88 \pm 5.31$ & 0.58 \\
\hline Daily gain, kg & $0.89 \pm 0.07$ & $0.98 \pm 0.05$ & 0.31 \\
\hline Height, $\mathrm{cm}$ & $93.43 \pm 2.63$ & $93.51 \pm 2.10$ & 0.98 \\
\hline Length, cm & $92.15 \pm 2.47$ & $92.61 \pm 3.78$ & 0.92 \\
\hline Chest girth, $\mathrm{cm}$ & $108.60 \pm 0.98$ & $108.45 \pm 1.49$ & 0.79 \\
\hline Daily intake, g & $373.47 \pm 95.41$ & $290.74 \pm 73.11$ & 0.52 \\
\hline
\end{tabular}


probiotics, there were no significant effects on daily weight gain, body height, body length, chest circumference and feed intake of the calves during the test period $(\mathrm{P}>0.05)$.

\subsection{Serum Index Results}

Table 3 shows the changes in the calf serum index for each group. Due to individual differences among the test animals, the calf serum index (Supplemental Table S1) between the groups before the test was statistically different, and this difference could not be avoided. Therefore, the change in serum results was statistically analyzed. The numerical magnitude represents the amount of change at two points in time, with positive and negative values indicating increases or decreases, respectively. The first stage is from 7 days to 15 days of age, indicating changes in serum indicators after treatment; the second stage is from 15 days to 63 days of age, indicating treatment for a period of time until the serum index changes after weaning. It can be seen from the table that the supplementation of

Table 3. Effects of probiotics on serum of calves.

\begin{tabular}{|c|c|c|c|}
\hline \multicolumn{3}{|c|}{ Group } & \multirow{2}{*}{$\mathrm{p}$} \\
\hline & $\mathrm{C}$ & $\mathrm{P}$ & \\
\hline \multicolumn{4}{|l|}{ First stage } \\
\hline $\operatorname{IgA}, \mu \mathrm{g} / \mathrm{mL}$ & $30.85 \pm 2.07$ & $33.82 \pm 2.11$ & 0.35 \\
\hline $\mathrm{IgG}, \mu \mathrm{g} / \mathrm{mL}$ & $-104.69 \pm 9.76$ & $-112.26 \pm 8.67$ & 0.58 \\
\hline $\mathrm{IL}-1 \beta, \mathrm{ng} / \mathrm{mL}$ & $17.82 \pm 1.74$ & $11.98 \pm 2.14$ & 0.08 \\
\hline TNF- $\alpha, \mathrm{ng} / \mathrm{mL}$ & $3.33 \pm 5.62$ & $3.63 \pm 2.67$ & 0.96 \\
\hline IL-4, ng/L & $47.93 \pm 1.98$ & $0.00 \pm 0.79$ & $<0.01$ \\
\hline IL-6, ng/L & $2.98 \pm 0.53$ & $2.51 \pm 0.16$ & 0.42 \\
\hline $\mathrm{IFN}-\gamma, \mathrm{ng} / \mathrm{L}$ & $-280.13 \pm 33.20$ & $-224.76 \pm 22.38$ & 0.22 \\
\hline $\mathrm{GH}, \mu \mathrm{g} / \mathrm{L}$ & $-1.08 \pm 0.43$ & $-3.61 \pm 0.28$ & $<0.01$ \\
\hline $\mathrm{LP}, \mu \mathrm{g} / \mathrm{L}$ & $-0.40 \pm 0.18$ & $1.48 \pm 0.14$ & $<0.01$ \\
\hline \multicolumn{4}{|l|}{ Second stage } \\
\hline $\mathrm{IgA}, \mu \mathrm{g} / \mathrm{mL}$ & $2.455 \pm 1.23$ & $0.70 \pm 0.47$ & 0.23 \\
\hline $\mathrm{IgG}, \mu \mathrm{g} / \mathrm{mL}$ & $-1.89 \pm 57.51$ & $65.45 \pm 9.68$ & 0.29 \\
\hline $\mathrm{IL}-1 \beta, \mathrm{ng} / \mathrm{mL}$ & $9.46 \pm 2.64$ & $10.60 \pm 2.85$ & 0.78 \\
\hline TNF- $\alpha, \mathrm{ng} / \mathrm{mL}$ & $9.09 \pm 5.07$ & $9.17 \pm 3.15$ & 0.99 \\
\hline $\mathrm{IL}-4, \mathrm{ng} / \mathrm{L}$ & $-41.86 \pm 1.29$ & $-45.35 \pm 4.54$ & 0.49 \\
\hline IL-6, ng/L & $7.31 \pm 0.45$ & $7.46 \pm 0.08$ & 0.76 \\
\hline IFN- $\gamma, \mathrm{ng} / \mathrm{L}$ & $153.00 \pm 20.55$ & $108.35 \pm 28.19$ & 0.25 \\
\hline $\mathrm{GH}, \mu \mathrm{g} / \mathrm{L}$ & $-0.12 \pm 0.17$ & $0.71 \pm 0.47$ & 0.15 \\
\hline $\mathrm{LP}, \mu \mathrm{g} / \mathrm{L}$ & $0.08 \pm 0.34$ & $0.90 \pm 0.27$ & 0.11 \\
\hline
\end{tabular}


probiotics only had significant effects on serum IL-4, GH and LP in the first stage $(\mathrm{P}<0.05)$, and had no significant effect on other serum indicators in the first stage or the second stage $(\mathrm{P}>0.05)$.

\subsection{S rDNA Sequencing Results}

From Figure 1, we found that the dilution curves of all samples tend to be flat, indicating that the sample is adequately sequenced and the depth has covered almost all species in the sample.

The tags were clustered at the $97 \%$ similarity level to obtain OTU, and OTU was classified based on the Silva (bacteria) taxonomy database to obtain the OTU number of each sample. A total of 349 OTUs were obtained from 8 samples. There are 337 OTUs in the control group and 302 OTUs in the P group. It can be seen from the Venn diagram that there are 290 OTUs in common among the two groups, 47 OTUs in only the C group, and 12 OTUs in only the P group (Figure 2).

The OTU representative sequence is compared with the microbial reference database to obtain species classification information corresponding to each OTU. Furthermore, the composition of each sample community was counted at each level (phylum, class, order, family, genus, species), and the species abundance at different classification levels was obtained. Only the top ten species in abundance levels are shown, and other species are combined into "Others". In the figure, Unclassified represents species that are not taxonomically annotated.

At the phylum level, 11 phyla were detected in group C rumen fluid, and 11 phyla were detected in group P (Figure 3). Group C mainly contained $49.95 \%$ of Bacteroidetes, 32.06\% of Firmicutes, 13.19\% of Proteobacteria, 1.64\% of Fibrobacteres, and $2.31 \%$ of Teneriquets. The P group mainly contained $43.90 \%$ of Bacteroidetes, $37.04 \%$ of Firmicutes, $17.79 \%$ of Proteobacteria.

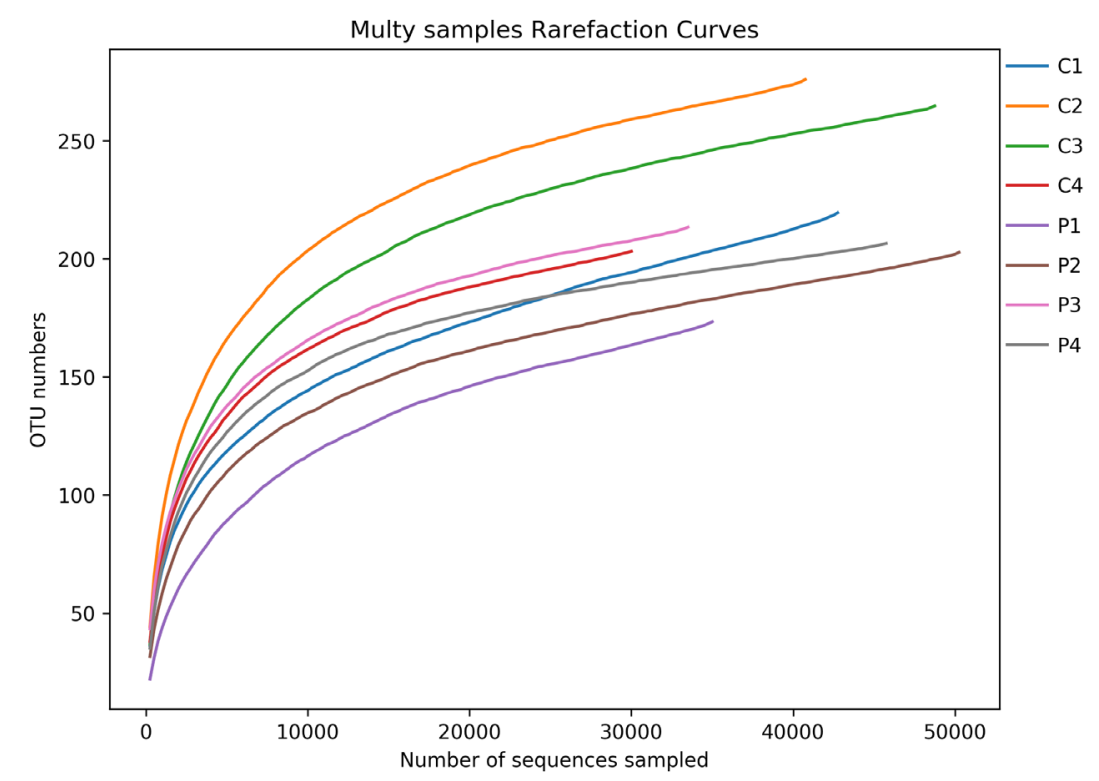

Figure 1. Sample-based rarefaction curve of observed species. 

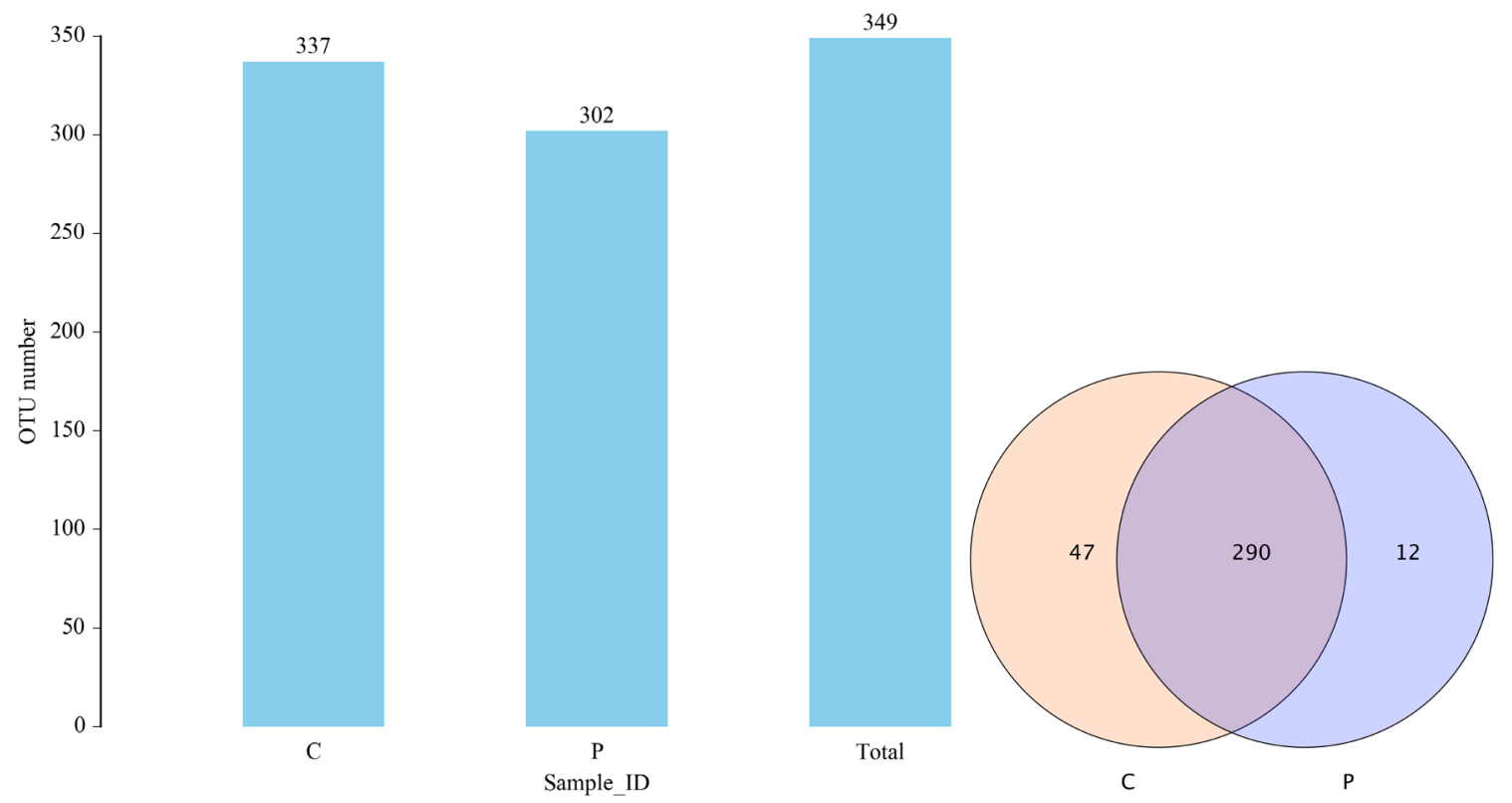

Figure 2. Shared OTUs across different groups.

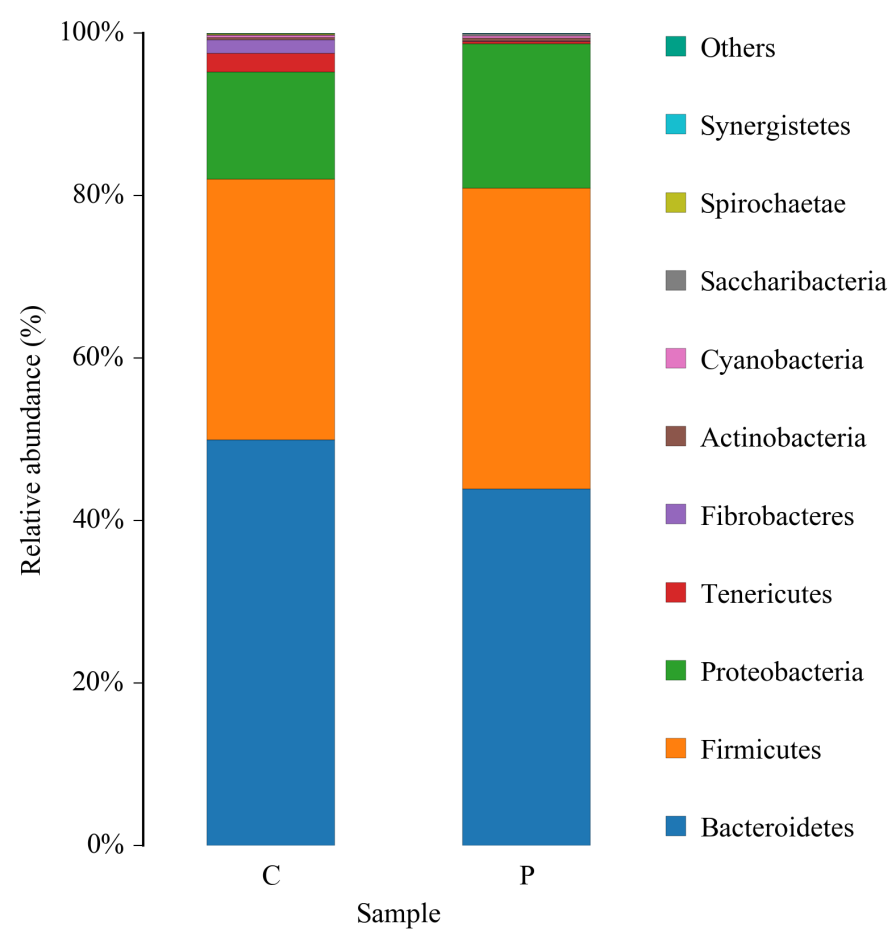

Figure 3. Taxonomic composition distribution in samples at the phylum level.

In Figure 4, at the genus level, 122 genera were detected in the rumen fluid of group C, and 121 genera were detected in rumen fluid in group P. Group C mainly contained $15.40 \%$ of Prevotella_7, 24.22\% of Prevotella_1, $10.93 \%$ of Succinivibrionaceae_UCG-001, 9.80\% of Roseburia. The P group mainly contained $19.04 \%$ of Prevotella_7, 18.85\% of Prevotella_1, 14.60\% of Succinivibrionaceae_UCG-001, 9.51\% of Roseburia, and 7.89\% of Megacphaera. 


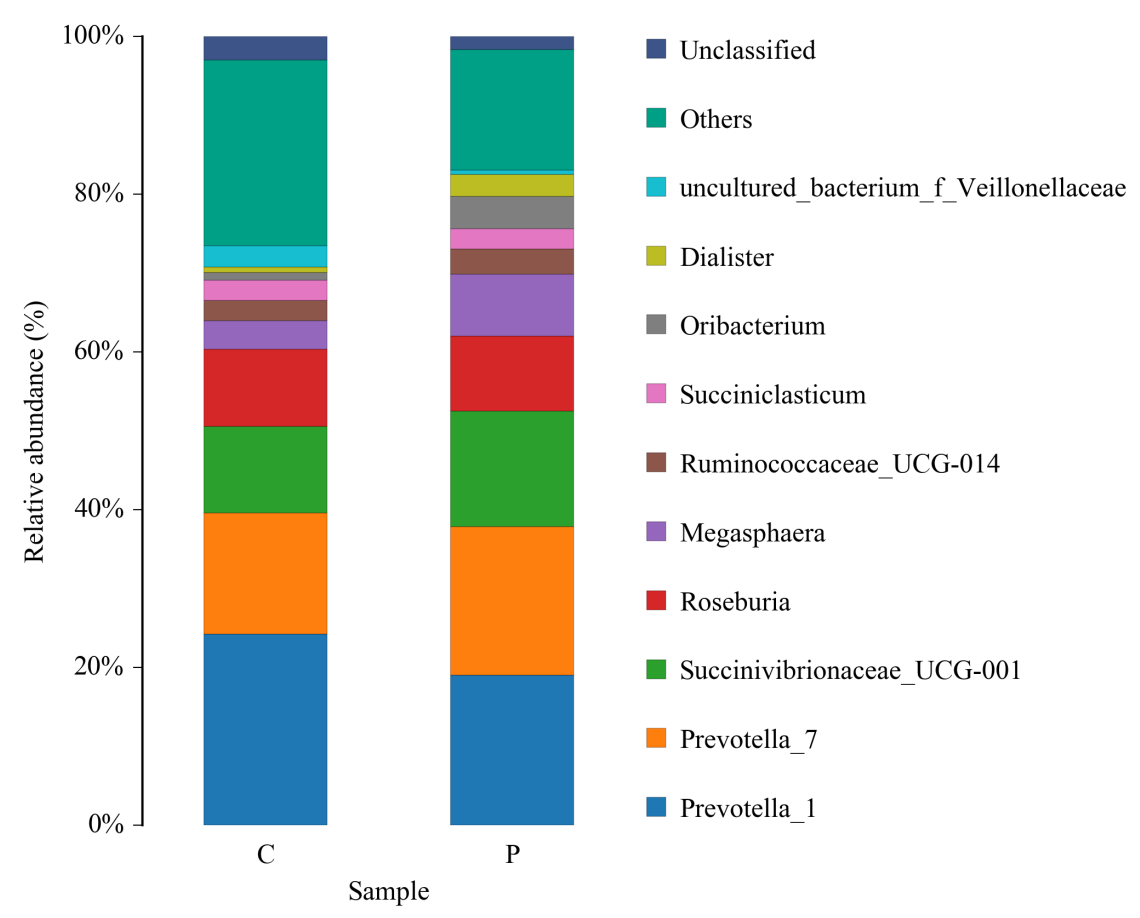

Figure 4. Taxonomic composition distribution in samples at the genus level.

Table 4 shows the taxonomic statistics of bacteria with rumen fluid bacteria content greater than $0.01 \%$ in groups $C$ and $\mathrm{P}$, among which, there were significant differences between Deltaproteobacteria, Desulfovibrionales, Bacteroidales BS11_gut_group, Desulfovibrionaceae, Bacteroidales_S24-7_group, Acetobacteraceae, Ruminococcaceae_NK4A214_group, Asaia, [Ruminococcus] gauvreauii_ group, Desulfovibrio, Kingella, Selenomonas, and Lachnoclostridium. There were different trends in Rhodospirillales, Burkholderiaceae, Veillonellaceae_UCG001, Succinivibrio, Megasphaera, Burkholderia-Paraburkholderia, and Ruminiclostridium_6.

\subsection{Metabolomics Analysis}

The differences in the level of metabolites between the two groups, as well as the statistical significance of these differences, can be quickly viewed through the volcano map. The differential expression volcano is as follows: Compared with group C, group P had 26 significant metabolites in positive ion mode, of which 22 were significantly decreased and 4 were significantly increased; There were 24 significant metabolites in negative ion mode, of which 7 was significantly decreased and 17 were significantly increased (Figure 5).

As can be seen from Table 5, compared with group C, some metabolites in group $\mathrm{P}$ were significantly increased, including 2-methylbenzoic acid, myo-inositol. The main related metabolic pathways include ascorbate and aldarate metabolism (ko00053), galactose metabolism (ko00052), inositol phosphate metabolism (ko00562). Significantly decreased metabolites include: thiamine, and the major metabolic pathways involved is thiamine metabolism (ko00730). 

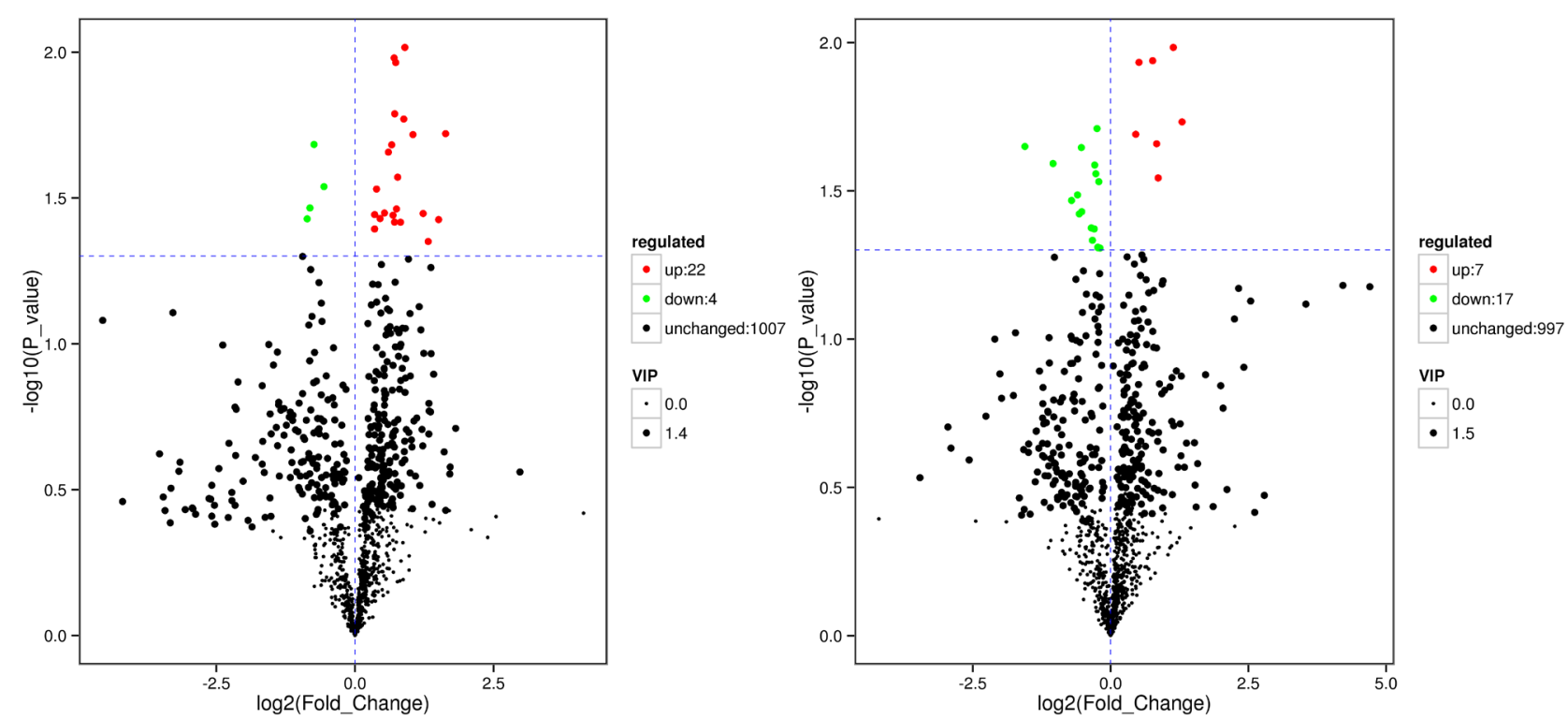

Figure 5. Volcano map of metabolite distribution between groups $\mathrm{C}$ and P. Notes: Each point in the volcano map represents a metabolite, the abscissa represents the fold change of the group compared to the substance (logarithm base 2), and the ordinate represents the P-value of the student's t test (logarithm base 10). The scatter size represents the VIP value of the OPLS-DA model. The larger the scatter, the larger the VIP value, and the more reliable the differentially expressed metabolites are. The green dots in the figure represent differentially expressed metabolites, the red dots represent up-regulated differentially expressed metabolites, and the black represents metabolites detected but not significantly different.

Table 4. The differences in bacterial contents of rumen fluid.

\begin{tabular}{|c|c|c|c|c|c|}
\hline Bacterium & Mean $(\mathrm{C})$ & Std.err (C) & Mean $(\mathrm{P})$ & Std.err (P) & $\mathrm{P}$ \\
\hline \multicolumn{6}{|c|}{ Class } \\
\hline Deltaproteobacteria & 1.34 & 0.33 & 0.39 & 0.18 & 0.03 \\
\hline \multicolumn{6}{|c|}{ Order } \\
\hline Desulfovibrionales & 1.29 & 0.30 & 0.38 & 0.17 & 0.02 \\
\hline Rhodospirillales & 0.02 & 0.00 & 0.06 & 0.02 & 0.08 \\
\hline \multicolumn{6}{|c|}{ Family } \\
\hline Bacteroidales_BS11_gut_group & 0.07 & 0.03 & 0.00 & 0.00 & 0.01 \\
\hline Desulfovibrionaceae & 1.29 & 0.30 & 0.38 & 0.02 & 0.02 \\
\hline Bacteroidales_S24-7_group & 0.06 & 0.20 & 0.09 & 0.07 & 0.03 \\
\hline Acetobacteraceae & 0.01 & 0.00 & 0.04 & 0.01 & 0.04 \\
\hline Burkholderiaceae & 0.01 & 0.00 & 0.02 & 0.01 & 0.09 \\
\hline \multicolumn{6}{|c|}{ Genus } \\
\hline Ruminococcaceae_NK4A214_group & 0.16 & 0.03 & 0.05 & 0.01 & $<0.01$ \\
\hline Asaia & 0.00 & 0.00 & 0.03 & 0.01 & $<0.01$ \\
\hline [Ruminococcus] gauvreauii_group & 0.19 & 0.05 & 0.02 & 0.01 & 0.01 \\
\hline Desulfovibrio & 1.29 & 0.30 & 0.38 & 0.17 & 0.01 \\
\hline Kingella & 0.01 & 0.00 & 0.02 & 0.00 & 0.03 \\
\hline Selenomonas & 2.06 & 0.75 & 0.33 & 0.06 & 0.03 \\
\hline
\end{tabular}


Continued

\begin{tabular}{cccccc}
\hline Lachnoclostridium & 0.01 & 0.00 & 0.04 & 0.02 & 0.03 \\
Veillonellaceae_UCG-001 & 0.14 & 0.07 & 0.00 & 0.00 & 0.07 \\
Succinivibrio & 0.42 & 0.34 & 2.29 & 1.02 & 0.08 \\
Megasphaera & 3.49 & 1.30 & 7.68 & 2.04 & 0.09 \\
Burkholderia-Paraburkholderia & 0.01 & 0.00 & 0.02 & 0.01 & 0.09 \\
Ruminiclostridium_6 & 0.01 & 0.00 & 0.00 & 0.00 & 0.10 \\
\hline
\end{tabular}

Table 5. Metabolites and their corresponding metabolic pathways.

\begin{tabular}{ccccc}
\hline Metabolite & Metabolic pathway & Fold-change & P & VIP \\
\hline 2-Methylbenzoic acid & Metabolic pathways (ko01100) & 1.373 & 0.020 & 2.156 \\
& $\begin{array}{c}\text { Ascorbate and aldarate metabolism (ko00053); } \\
\text { Galactose metabolism (ko00052); } \\
\text { Myo-Inositol } \\
\text { Inositol phosphate metabolism (ko00562) } \\
\text { Thiamine }\end{array}$ & 1.429 & 0.012 & 2.315 \\
Thiamine metabolism (ko00730) & 0.485 & 0.026 & 2.123 \\
\hline
\end{tabular}

\section{Discussion}

\subsection{Effect of Supplemental Probiotics on the Growth of Calves}

In the experiment, the supplemental probiotics only increased the daily weight gain of calves, but the difference was not significant $(\mathrm{P}>0.05)$. Feeding probiotics reduced the feed intake of calves by numerical value, and the difference was not significant $(\mathrm{P}>0.05)$. Experiments with yeast or yeast cultures fed to ruminants also have inconsistent results. Studies have found no significant changes in body weight or even reduced body weight after feeding [22]. Studies have also found that weight gain significantly increases after feeding and feed utilization is improved. Kawas mentioned that supplemental yeast improved the weight gain of lambs fed low-protein diets and did not have any beneficial effect on lambs fed high-protein diets. There was no significant difference in body weight and feed efficiency among Holstein calves fed with Bacillus subtilis cultures [23]. There is a lack of consistent results on calves fed with probiotics, which have been shown to improve growth performance, increase body weight and feed conversion, and have also been shown to have no benefit. The results of the researchers' studies on probiotics are not completely consistent. The results of this experiment show that the feeding of probiotics can improve the growth performance of calves to some extent, but the effect is not significant.

\subsection{Effect of Supplemental Probiotics on Calf Serum}

IL-4 is a lymphoid factor secreted by $\mathrm{T}$ cells with a variety of biological functions. It has a proliferative differentiation effect on B cells under the synergistic action of other cytokines. In the control group, IL-4 was significantly increased in the first stage, but there was no significant change in the P group. Studies have shown that feeding Bacillus subtilis has no effect on serum IgA and IL-6 of 
calves, but an increase occurrs in IFN- $\gamma$ [24]. In this experiment, the effect of feeding probiotics on calf serum can only be shown with individual serum indicators, but the specific mechanism needs further research.

\subsection{Effect of Supplemental Probiotics on Microbes in Rumen Fluid of Calves}

Feeding starter to unweaned lambs significantly increased the abundance of unclassified_BS11_gut_group in the rumen [25]. Adding monensin significantly reduced the abundance of cow Bacteroidales_BS11_gut_group and Ruminococcaceae [26]. Bacteroidales_BS11_gut_group was not detected in group P; thus, the abundance was significantly lower than that of group $C$. The abundance of Ruminococcaceae_NK4A214_group, [Ruminococcus]_gauvreauii_group, and Ruminiclostridium_6 were also significantly lower in group P than in group C, which was consistent with the result of adding monensin. The addition of daidzein significantly reduced the abundance of Bacteroidales_S24-7_group in calf feces [27], while the abundance of Bacteroidales_S24-7_group was significantly higher with probiotics in group $\mathrm{P}$ than in group $\mathrm{C}$ in our trial. Wheat germ globulin can increase the number of beneficial bacteria in the intestine and maintain the dynamic balance of intestinal microflora. The abundance of Ruminococcaceae NK4A214 group in wheat germ globulin model mice is reduced [28]. Wheat bran feeding piglets can reduce the proliferation of intestinal pathogens by reducing the amount of digestion. After feeding wheat bran, the abundance of Ruminococcaceae NK4A214 group is reduced in pig feces. In the experiment, the abundance of Ruminococcaceae NK4A214 group in group P was significantly lower than that in group C, which was consistent with the results in other studies. In 2008, Domingo and others isolated the anti-glycation type Ruminococcus gauvreauii from human feces, which is a strictly anaerobic gram-positive cocci and is significantly increased in the intestinal tract of diabetic rats [29]. The abundance of Ruminococcus gauvreauii in group P was significantly lower than that in group C. Studies have shown that anaerobic bacteria produce active phytase in ruminants, especially Selenomonas [30]. Under the stimulation of sacchariae culture, the utilization of lactic acid by Selenomonas can be improved [31]. In this study, Selenomonas in group $\mathrm{P}$ was significantly lower than that in group C. Thus, we demonstrate that supplemental feeding of probiotics can change the abundance of some bacteria to a direction more conducive to the growth needs of calves on the basis of maintaining normal rumen bacteria.

\subsection{Effect of Supplemental Probiotics on Rumen Fluid and Metabolic Pathways in Calves}

We found that supplemental probiotics only have significant effects on a small number of metabolites in the rumen fluid of calves. Among them, the metabolites myo-inositol and 2-methylbenzoic acid, which relate to the carbohydrate metabolism pathway, were significantly increased. Vitamin B1, which is associated with the cofactors and vitamins metabolism pathways, is significantly re- 
duced. Inositol, also known as cyclohexanol, is a bioactive sugar alcohol and a growth factor of animals and microorganisms. The main microorganisms producing inositol are Saccharomyces cerevisiae, Escherichia coli and Bacillus subtilis [32]. The metabolic pathways of inositol have been found in many bacteria, and most of the genes involved in these pathways are conserved [33]. Studies have shown that Rhizobium leguminosarum bv. viciae and Sinorhozobium meliloti can utilize the abundant inositol in soil as the sole source of carbon for growth [34]. Supplementation of probiotics increases the levels of a few metabolites in rumen fluid and may promote the pathway of carbohydrate metabolism.

\section{Conclusion}

In this study, the effects of active probiotics on the growth performance of calves and the colonization of rumen bacteria were studied. It was found that the supplemental active probiotics had an effect on the rumen microflora, but had little effect on growth performance and metabolites.

\section{Acknowledgements}

The study was financially supported by the earmarked fund for the Key R \& D plan of shandong province (2019JZZY010704) and thanks for the cooperation with Shandong Bilan Biological Technology Company.It was also supported by the National Key R \& D program of China (2017YFD0500502), Cattle Agro-industry Technology Research System of Shandong Province (SDAIT-12-011-06), Natural Science Fund of China (31572427) (31372340) and Taishan scholar project.

\section{Conflicts of Interest}

The authors declare no conflicts of interest regarding the publication of this paper.

\section{References}

[1] Metchnikoff, E. (1908) The Prolongation of Life. GP Putman's Sons Publishers, New York.

[2] Lilly, D. and Stillwell, R. (1965) Probiotics-Growth-Promoting Factors Produced by Microorganisms. Science, 147, 747-748. https://doi.org/10.1126/science.147.3659.747

[3] Parker, R.B. (1974) Probiotics, the Other Half of Antibiotic Story. Animal Nutrition \& Health, 29, 4-8.

[4] Antunovic, Z., Speranda, M., Liker, B., et al. (2005) Influence of Feeding the Probiotic Pioneer PDFM (R) to Growing Lambs on Performances and Blood Composition. Acta Veterinaria, 55, 287-300. https://doi.org/10.2298/AVB0504287A

[5] Mountzouris, K.C., Balaskas, C., Xanthakos, I., et al. (2009) Effects of a Multi-Species Probiotic on Biomarkers of Competitive Exclusion Efficacy in Broilers Challenged with Salmonella Enteritidis. British Poultry Science, 50, 467-478. https://doi.org/10.1080/00071660903110935 
[6] Musa, H., Wu, S., Zhu, C., et al. (2009) The Potential Benefits of Probiotics in Animal Production and Health. Journal of Animal and Veterinary Advances, 8, 313321.

[7] Krehbiel, C., Rust, S., Zhang, G. and Gilliland, S. (2003) Bacterial Direct-Fed Microbials in Ruminant Diets: Performance Response and Mode of Action. Journal of Animal Science, 81, E120-E132.

[8] Bomba, A., Nemcová, R., Gancarcíková, S., et al. (2002) Improvement of the Probiotic Effect of Micro-Organisms by Their Combination with Maltodextrins, Fructo-Oligosaccharides and Polyunsaturated Fatty Acids. British Journal of Nutrition, 88, S95-S99. https://doi.org/10.1079/BJN2002634

[9] Isolauri, E., Sutas, Y., Kankaanpaa, P., et al. (2001) Probiotics: Effects on Immunity. American Journal of Clinical Nutrition, 73, 444S-450S.

https://doi.org/10.1093/ajcn/73.2.444s

[10] Perdigon, G., Alvarez, S., Rachid, M., et al. (1995) Immune System Stimulation by Probiotics. Journal of Dairy Science, 78, 1597-1606. https://doi.org/10.3168/jds.S0022-0302(95)76784-4

[11] Matsuzaki, T. and Chin, J. (2000) Modulating Immune Responses with Probiotic Bacteria. Immunology and Cell Biology, 78, 67-73. https://doi.org/10.1046/j.1440-1711.2000.00887.x

[12] Roselli, M., Finamore, A., Britti, M., et al. (2005) Alternatives to In-Feed Antibiotics in Pigs: Evaluation of Probiotics, Zinc or Organic Acids as Protective Agents for the Intestinal Mucosa: A Comparison of in vitro and in vivo Results. Animal Research, 54, 203-218. https://doi.org/10.1051/animres:2005012

[13] Guillot, J.F. (2003) Probiotic Feed Additives. Journal of Veterinary Pharmacology and Therapeutics, 26, 52-55.

[14] Casas, I.A. and Dobrogosz, W.J. (2000) Validation of the Probiotic Concept: Lactobacillus Reuteri Confers Broad-Spectrum Protection against Disease in Humans and Animals. Microbial Ecology in Health and Disease, 12, 247-285.

https://doi.org/10.1080/08910600050216246-1

[15] Yuan, X.P., Wang, J. and Yao, H.Y. (2004) Purification and Some Properties of Endoxylanases from Bacillus subtilis. Food and Fermentation Industries, 30, 55-59.

[16] Fan, Y.J., Pang, W., Shi, H.Y., et al. (2009) Preliminary Study on Fermentation Technology of Swine Blood Protein Peptide with Bacillus subtili. Journal of Anhui Agricultural Sciences, 37, 7184-7186.

[17] Li, H.F., Ye, Y.H. and Guo, J.H. (2010) Isolation and Identification of Bacillus subtilis 7Ze3 Cyclic Dipeptide. Jiangsu Journal of Agricultural Sciences, 2, 107-109.

[18] Zhang, L., Zhang, D., Zhang, L.P., et al. (2010) Optimization of Fermentation Medium for Production of Antibacterial Peptides by Bacillus subtilis BSD-2. Food Science, 31, 189-192.

[19] Ding, X.J., Zhang, X.T., Wang, S.Q., et al. (2017) Effects of Saccharomyces cerevisiae Culture on Growth Performance, Apparent Availability of Nutrients and Intestinal Bacteria Flora of 817 Broiler Chickens. Chinese Journal of Animal Nutrition, 29, 2391-2398.

[20] Bruno, R.G.S., Rutigliano, H.M., et al. (2009) Effect of Feeding Saccharomyces cerevisiae on Performance of Dairy Cows during Summer Heat Stress. Animal Feed Science and Technology, 150, 175-186. https://doi.org/10.1016/j.anifeedsci.2008.09.001

[21] Williams, P., Tait, C., Innes, G., et al. (1991) Effects of the Inclusion of Yeast Cul- 
ture (Saccharomyces cerevisiae plus Growth Medium) in the Diet of Dairy-Cows on Milk-Yield and Forage Degradation and Fermentation Patterns in the Rumen of Steers. Journal of Animal Science, 69, 3016-3026.

https://doi.org/10.2527/1991.6973016x

[22] Kawas, J.R., García-Castillo, R., Garza-Cazares, F., et al. (2007) Effects of Sodium Bicarbonate and Yeast on Productive Performance and Carcass Characteristics of Light-Weight Lambs Fed Finishing diets. Small Ruminant Research, 67, 157-163. https://doi.org/10.1016/j.smallrumres.2005.09.011

[23] Jenny, B.F., Vandijk, H.J. and Collins, J.A. (1991) Performance and Fecal Flora of Calves Fed a Bacillus subtilis Concentrate. Journal of Dairy Science, 74, 1968-1973. https://doi.org/10.3168/jds.S0022-0302(91)78364-1

[24] Sun, P., Wang, J.Q. and Zhang, H.T. (2011) Effects of Bacillus subtilis natto on Performance and Immune Function of Preweaning Calves. Journal of Dairy Science, 93, 5851-5855. https://doi.org/10.3168/jds.2010-3263

[25] Liu, J., Bian, G., Sun, D., et al. (2017) Starter Feeding Altered Ruminal Epithelial Bacterial Communities and Some Key Immune-Related Genes' Expression before Weaning in Lambs 1. Journal of Animal Science, 95, 910.

[26] Scharen, M., Drong, C., Kiri, K., et al. (2017) Differential Effects of Monensin and a Blend of Essential Oils on Rumen Microbiota Composition of Transition Dairy Cows. Journal of Dairy Science, 100, 2765-2783. https://doi.org/10.3168/jds.2016-11994

[27] Zhao, X.H., Chen, Z.D., Zhou, S., et al. (2017) Effects of Daidzein on Performance, Serum Metabolites, Nutrient Digestibility, and Fecal Bacterial Community in Bull Calves. Animal Feed Science and Technology, 225, 87-96. https://doi.org/10.1016/j.anifeedsci.2017.01.014

[28] Jiao, X.G. (2018) Proteomic Analysis of Wheat Germ Globulin and Its Effects on Intestinal Microfloras. Henan University of Technology, Zhengzhou.

[29] Wang, P.L. (2015) The Effects of Liraglutide on Diabetic Rat Intestinal Flora and Its Metabolites. Peking Union Medical College, Beijing.

[30] Yanke, L.J., Bae, H.D., Selinger, L.B., et al. (1998) Phytase Activity of Anaerobic Ruminal Bacteria. Microbiology, 144, 1565-1573.

https://doi.org/10.1099/00221287-144-6-1565

[31] Nisbet, D. and Martin, S. (1991) Effect of a Saccharomyces-Cerevisiae Culture on Lactate Utilization by the Ruminal Bacterium Selenomonas-Ruminantium. Journal of Animal Science, 69, 4628-4633. https://doi.org/10.2527/1991.69114628x

[32] Yamaoka, M., Osawa, S., Morinaga, T., et al. (2011) A Cell Factory of Bacillus subtilis Engineered for the Simple Bioconversion of Myo-Inositol to Scyllo-Inositol, a Potential Therapeutic Agent for Alzheimer's Disease. Microbial Cell Factories, 10, Article No. 69. https://doi.org/10.1186/1475-2859-10-69

[33] Yebra, M.J., Zúñiga, M., Beaufils, S., et al. (2007) Identification of a Gene Cluster Enabling Lactobacillus casei BL23 to Utilize Myo-Inositol. Applied and Environmental Microbiology, 73, 3850-3858. https://doi.org/10.1128/AEM.00243-07

[34] Bahar, M., de Majnik, J., Wexler, M., et al. (1998) A Model for the Catabolism of Rhizopine in Rhizobium leguminosarum Involves a Ferredoxin Oxygenase Complex and the Inositol Degradative Pathway. Molecular Plant-Microbe Interactions, 11, 1057-1068. https://doi.org/10.1094/MPMI.1998.11.11.1057 


\section{Supplemental}

Supplemental Table S1. Serum indicators of calves at 7 days of age.

\begin{tabular}{cccc}
\hline & Group & & P \\
\hline IgA, $\mu \mathrm{C} / \mathrm{mL}$ & $41.04 \pm 1.13$ & $\mathrm{P}$ & 0.38 \\
$\mathrm{IgG}, \mu \mathrm{g} / \mathrm{mL}$ & $559.05 \pm 10.87$ & $560.07 \pm 5.38$ & 0.94 \\
$\mathrm{IL}-1 \beta, \mathrm{ng} / \mathrm{mL}$ & $49.22 \pm 2.51$ & $45.66 \pm 0.72$ & 0.22 \\
$\mathrm{TNF}-\alpha, \mathrm{ng} / \mathrm{mL}$ & $52.18 \pm 1.66$ & $50.55 \pm 1.01$ & 0.43 \\
$\mathrm{IL}-4, \mathrm{ng} / \mathrm{L}$ & $58.58 \pm 1.69$ & $107.53 \pm 1.32$ & $<0.01$ \\
$\mathrm{IL}-6, \mathrm{ng} / \mathrm{L}$ & $13.30 \pm 0.59$ & $13.10 \pm 0.18$ & 0.76 \\
$\mathrm{IFN}-\gamma, \mathrm{ng} / \mathrm{L}$ & $749.93 \pm 27.41$ & $788.33 \pm 21.83$ & 0.32 \\
$\mathrm{GH}, \mu \mathrm{g} / \mathrm{L}$ & $15.24 \pm 0.19$ & $15.98 \pm 0.09$ & 0.01 \\
$\mathrm{LP}, \mu \mathrm{g} / \mathrm{L}$ & $6.68 \pm 0.11$ & $5.53 \pm 0.13$ & $<0.01$ \\
\hline
\end{tabular}

